\title{
Paced segment characteristics predict clinical response to cardiac resynchronization therapy: results from the multimodality imaging assessment of pacing intervention in heart failure (MAPIT-HF) study
}

\author{
Jorge A Wong*, David Scholl, Raymond Yee, John Stirrat, Kris Carter, David McCarty, Nowell Fine, Andrew Krahn, \\ Lorne Gula, Allan Skanes, Peter Leong-Sit, George Klein, Maria Drangova, James A White
}

From 2011 SCMR/Euro CMR Joint Scientific Sessions

Nice, France. 3-6 February 2011

\section{Introduction}

Cardiac Resynchronization Therapy (CRT) has been shown to improve quality of life and decrease mortality in heart failure patients. However, up to $40 \%$ of patients fail to respond to this therapy. Validation of a response prediction model that incorporates both myocardial scar and dyssynchrony of the paced myocardial segments would allow for a targeted approach to CRT lead delivery.

\section{Methods}

Patients planned for CRT under standard indications were prospectively enrolled. Serial short-axis tagged cine and delayed enhancement MRI was performed using standard imaging protocols. Echocardiography and gated CT Angiography (CTA) were performed at baseline and 3 months post-implantation. The repeat CTA was performed for accurate lead tip localization to a 16-segment model. Dyssynchrony was measured for each segment (time to maximal radial strain, Trs) from serial shortaxis tagged MRI and expressed in milliseconds from onset of pulse trigger (InTag, OsirX). A segmental scar score was then assigned using a blinded visual interpretation (score 0 to 4 ). The number of response prediction rules met was determined for each patient as follows: 1) LV lead tip placed on a dyssynchronous segment (Trs > $130 \mathrm{msec}), 2) \mathrm{LV}$ lead tip placed on a viable segment (scar score <2), 3) RV lead tip placed on a viable segment (scar score $<2$ ). Clinical response to CRT, defined as a $>=15 \%$ reduction in LVESV by echocardiography, was correlated to the number of prediction rules met.

\section{Results}

Forty consecutive patients were enrolled with a mean age and ejection fraction of $67.0 \pm 8.6$ years and $25.6 \pm$ $6.6 \%$, respectively. Twenty four patients $(60 \%)$ met clinical response criteria with a mean reduction in LVESV of $20.5 \pm 16.5 \%$ compared to a rise of $1.4 \pm 6.3 \%$ in nonresponders $(\mathrm{p}<0.001)$. A strong correlation was seen between the number of prediction rules met and clinical response to CRT. In patients with 3, 2, 1 and 0 prediction rules met, the response rates were $100 \%, 92 \%, 58 \%$ and $40 \%$, respectively $(\mathrm{p}<0.001)$. A strong correlation was also seen between the number of prediction rules and the mean reduction in LVESV [28\%, $14 \%$ and 5\% reduction in those with 3,2 and 1 rules met, respectively $(\mathrm{p}=0.002)]$.

\section{Conclusions}

Dyssynchrony and scar characteristics of the paced myocardial segments are strongly correlated with clinical response to CRT. A simple 3-point prediction model incorporating these variables appears to be highly predictive of response, and may be valuable for the selection of optimal pacing targets for CRT.

University of Western Ontario, London, ON, Canada

( 2011 Wong et al; licensee BioMed Central Ltd. This is an open access article distributed under the terms of the Creative Commons 
doi:10.1186/1532-429X-13-S1-050

Cite this article as: Wong et al:: Paced segment characteristics predict clinical response to cardiac resynchronization therapy: results from the multimodality imaging assessment of pacing intervention in heart failure (MAPIT-HF) study. Journal of Cardiovascular Magnetic Resonance 2011 13(Suppl 1):050

Submit your next manuscript to BioMed Central and take full advantage of:

- Convenient online submission

- Thorough peer review

- No space constraints or color figure charges

- Immediate publication on acceptance

- Inclusion in PubMed, CAS, Scopus and Google Scholar

- Research which is freely available for redistribution 